\title{
Streptomycin Applications to Control Walnut Blight Disease Can Prevent Fertilization and Increase Fruit Drop
}

\author{
V.S. Polito ${ }^{1}$, K. Pinney ${ }^{2}$, R. Buchner ${ }^{3}$, and W. Olson ${ }^{3}$ \\ Department of Pomology, University of California, Davis, CA 95616
}

Additional index words. abscission, Agri-mycin, antibiotic, Juglans regia, ovule, pollen, pollination, Xanthomonas campestris pv. juglandis

\begin{abstract}
We investigated the basis for fruit drop in walnut (Juglans regia L.) following bloom period applications of streptomycin as a potential control treatment for walnut blight, a bacterial disease incited by Xanthomonas campestris pv. juglandis (Pierce) Dye. Experiments were conducted on streptomycin-treated field plots of 'Vina' walnut. Four streptomycin treatments were applied at different times relative to anthesis. Fruit from all treatments grew similarly for four weeks following anthesis when high levels of fruit abscission began to occur in the treatment sprayed during the bloom period. Microscopy revealed that in this treatment ovules failed to develop normally, and neither embryo nor endosperm developed. The pattern of fruit development and timing of fruit drop following streptomycin treatment at bloom is similar in all ways to that of unpollinated walnut flowers where growth appears normal until abscission occurs 3 to 5 weeks after anthesis. Pollen germination and pollen tube growth were inhibited in the bloom-period treatments. Pollen germination in vitro was not affected by addition of streptomycin to a germination medium. If streptomycin were to be used in a walnut blight control program, application timed to coincide with the period of pistillate bloom and pistillate flower receptivity should be avoided.
\end{abstract}

Blight disease of walnut (Juglans regia) is incited by the bacterium Xanthomonas campestris pv. juglandis. Teviotdale and Schroth (1998) describe the disease and summarize control measures. Bacteria invade a tree through stomata and other natural openings. The presence of free water is necessary for bacterial entry at any site. Fruits are susceptible to blight from flowering through the summer. In fruit that are infected prior to shell hardening, the kernel shrivels and the fruit often drops. Rainfall is the major contributor to disease spread and infection. Infection is negligible once the rainy season ends. In California walnut-growing areas, severity of losses to walnut blight increases in years with wet springs. Control practices are based on copper-containing materials which may be applied 1 to 10 times depending on the length of the rainy season. More frequent applications are used in northern California production areas characterized by high spring precipitation. There are no blight-resistant walnut cultivars. Bacteria tolerant of copper are increasingly common in some northern California walnut orchards, which has led to use of fungicides in conjunction with copper to enhance control (Teviotdale and Schroth, 1998).

Streptomycin, an aminoglycoside antibiotic, can be an effective control measure to reduce losses to walnut blight. Although it is

Received for publication 6 Aug. 2001. Accepted for publication 27 Nov. 2001.

${ }^{1}$ Professor. To whom reprint requests should be addressed. E-mail: vspolito@ucdavis.edu.

${ }^{2}$ Staff Research Associate.

${ }^{3}$ Cooperative Extension Adviser. not currently registered for use on walnuts, it has potential for use in northern Calif. walnut orchards where copper-resistant bacteria are common (Buchner et al., 2000). However, streptomycin applied during pistillate bloom can result in high incidence of fruit drop several weeks after the spray treatment. Our objective in this project was to determine the mechanism by which streptomycin applied during walnut bloom causes fruit drop.

\section{Materials and Methods}

Agri-mycin 17 (Novartis Crop Protection, Greensboro, N.C.), containing $22.4 \%$ streptomycin sulfate, was applied at $200 \mathrm{mg} \cdot \mathrm{L}^{-1}, 935$ $\mathrm{L} \cdot \mathrm{ha}^{-1}$ (100 gal per acre) by airblast backpack sprayer to a 'Vina' walnut orchard in Durham, Butte County, Calif. Four treatments were applied: one at the prebloom stage (6 Apr.), one during the bloom cycle $(14,21$, and 30 Apr.), one prebloom and postbloom (6 Apr. and 10 May) and one postbloom (10 May). The experiment was designed as a randomized complete block with four, single-tree replicates for each treatment and the control.

One hundred and fifty flowers were tagged on each treated tree at peak pistillate bloom. Set was determined by counting fruit remaining from the population of tagged flowers. On 21, 28 Apr. and 6, 13, and 20 May, eight flowers were randomly collected from each tree of the unsprayed control and each replicate that had received a treatment spray. For each collection, flowers were separated into ovary and stigma-style portions. Each portion was fixed separately in 3\% paraformaldehyde,
$50 \%$ ethanol, $5 \%$ acetic acid (FAA). The ovaries were dehydrated in a tert-butyl alcohol series and embedded in Paraplast Plus (Sherwood Medical, St. Louis). Ovaries were sectioned at a nominal thickness of $8 \mu \mathrm{m}$ and stained with safranin $\mathrm{O}$-fast green $\mathrm{FCF}$. The stigma-style portions of flowers from the prebloom, bloom-period and control treatments were softened, gently squashed on slides, stained with alkaline aniline blue, and observed with a fluorescence microscope. Number of pollen tubes present in the stigma and at the base of the style was recorded for 20 flowers for each treatment.

Pollen of walnut cultivars Hartley and Vina were germinated in vitro using the medium of Luza and Polito (1987) supplemented with $400,200,100,10$, and $0 \mathrm{mg} \cdot \mathrm{L}^{-1}$ streptomycin added to the medium after it had cooled to below $50{ }^{\circ} \mathrm{C}$. Three replicates were made for each cultivar at each concentration. Pollen was collected from catkins that were allowed to dehisce overnight. The pollen was hydrated in a saturated environment for $120 \mathrm{~min}$, then incubated on the medium for $3 \mathrm{~h}$ after which the plates were sprayed with FAA to halt pollen tube growth. Germination was determined for 100 pollen grains from each replicate. Pollen was considered to have germinated when pollen tube elongation was at least twice the diameter of the pollen grain. Pollen tube growth was not measured directly, but a visual estimation of comparative pollen tube elongation was made.

\section{Results}

At the macroscopic level, fruit from all treatments appeared to develop normally until fruits began to abscise in mid-May. Streptomycin application during the bloom period resulted in significantly increased fruit drop (Table 1). Examination of sectioned ovaries revealed that internal ovule tissues of fruit from the bloom-period streptomycin treatment had not developed normally. The 13 May samples showed marked differences at the micropylar ends of the ovules from the bloom-period treatment (Fig. 1A) relative to ovules from the control treatment (Fig. 1BC). Bloom-period treated ovules were about two-thirds the size of normal ovules. Tissue necrosis was evident at the apical region of the integument, and nucellar tissue had grown beyond the micropylar opening.

By 13 May, extensive endosperm development (Fig. 1, B) and early stages of embryo

Table 1. Fruit drop following streptomycin treatments of walnut (Juglans regia L. cv. Vina) at different stages of the bloom cycle. Values not followed by a common letter are significantly different from each other (Student-NewmanKeuls multiple comparison, $P=0.05$ ).

\begin{tabular}{lc}
\hline \hline Treatment & Fruit drop (\%) \\
\hline Bloom period & $89.5 \mathrm{~b}$ \\
Prebloom and postbloom & $32.4 \mathrm{a}$ \\
Postbloom & $10.6 \mathrm{a}$ \\
Unsprayed & $14.2 \mathrm{a}$ \\
Prebloom & $32.7 \mathrm{a}$ \\
\hline
\end{tabular}


growth (Fig. 1C) were evident in ovules from untreated controls. Endosperm and embryo development were lacking in the bloom-period treated ovules (Fig. 1A). At the chalazal end of these ovules (Fig. 1D), lacunae had begun to develop at the juncture of the chalaza and the funiculus, and nucellar tissue showed signs of degeneration.

Mean numbers of pollen tubes growing in the stigma and at the base of the style for prebloom, bloom-period and control treatments are shown in Fig. 2. Pollen tube numbers at the apical portions of the stigma and at the base of the style were reduced somewhat in the prebloom treatment relative to the untreated control. Bloom-period treated flowers showed reduction of pollen tubes at both locations. Pollen tubes were present at the style bases in all flowers from the prebloom treatment and the unsprayed control, but in only three of the 20 stigma-style squashes from the bloom-period treatment. Because these data are not normally distributed, treatments were compared using the Kruskal-Wallis test (Sokal and Rohlf, 1995). The differences among treatments are highly significant for median number of pollen tubes at the stigma $(\mathrm{H}=12.1,2 \mathrm{df}, P<0.002)$ and pollen tubes at the style base $(\mathrm{H}=27.9,2 \mathrm{df}, P<0.001)$. For both stigma and style, median pollen tubes are significantly different for each of these treatments (Student-Newman-Keuls multiple comparison, $P<0.05$ ).

Pollen tubes from the prebloom treatments and untreated controls grew normally in the stigmatic-stylar transmitting tissue (Fig. 3A). These pollen tubes are long, unbranched and have regular callose plugs. Pollen tubes from the bloom-period treatment (Fig. 3B-D) showed extensive

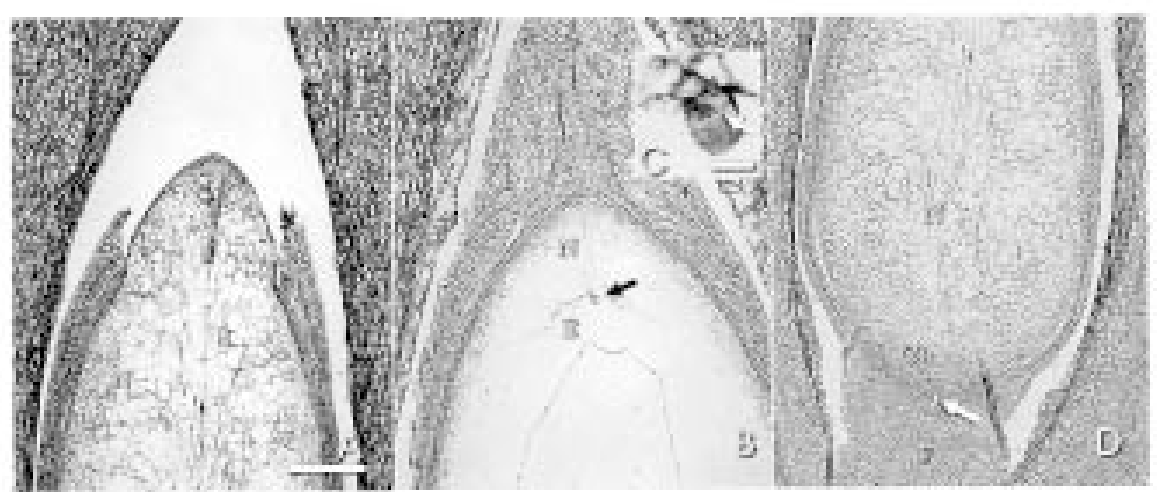

Fig. 1. Walnut ovules from 13 May. (A) Micropylar region of ovule from bloom-period treatment. (B) Micropylar region of ovule from untreated control. Arrow indicates the proembryo. (C) Proembryo shown at higher magnification. Note the first division of the zygote (arrow). (D) Chalzal region of ovule from bloom-period treatment. Arrow indicates lacuna forming at the funiculus-chalaza interface. $\mathrm{Ch}=$ chalaza; $\mathrm{E}=$ endosperm; $\mathrm{F}=$ funiculus; $\mathrm{I}=$ integument; $\mathrm{N}=$ nucellus. $(\mathbf{A}),(\mathbf{B})$ and $(\mathbf{D})$ are shown at same magnification; scale bar $=200 \mu \mathrm{m}$. (C) scale bar $=10 \mu \mathrm{m}$.

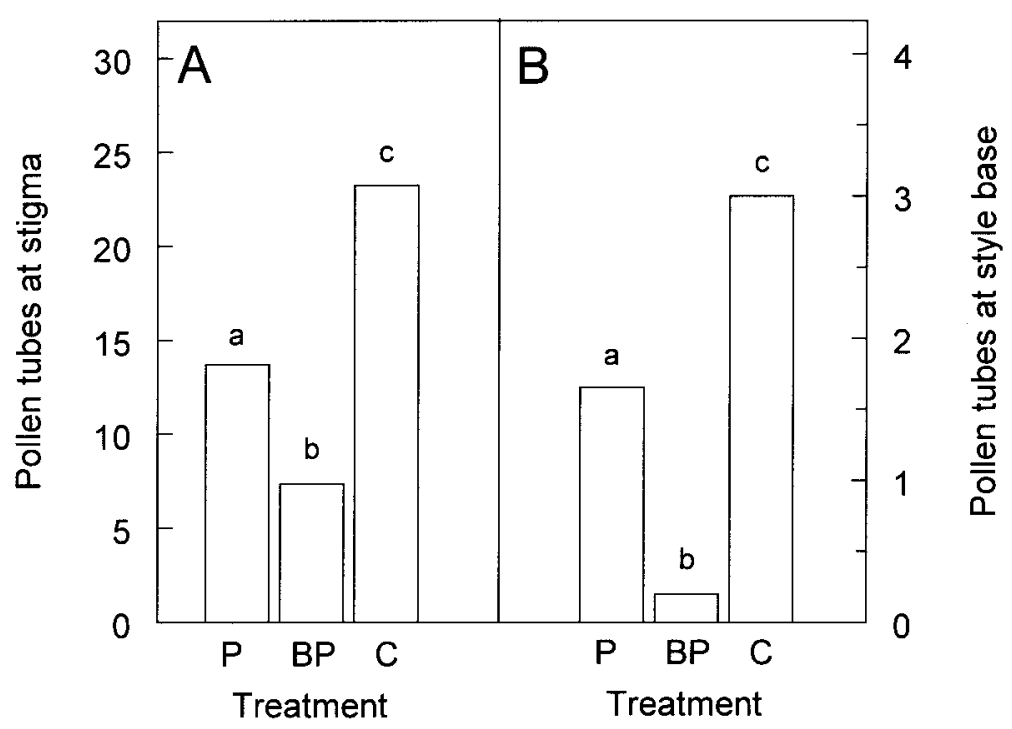

Fig. 2. Pollen tubes growing in the stigmas (A) and to the base of the styles $(\mathbf{B})$ of flowers from the prebloom streptomycin treatment $(\mathrm{P})$, bloom-period streptomycin treatment $(\mathrm{BP})$, and controls $(\mathrm{C}) . \mathrm{N}=20$ flowers for each treatment. The differences among treatments are highly significant for pollen tubes at the stigma and for pollen tubes at the style base (Kruskal-Wallis test, $P<0.01$ ). Treatment difference are noted by lower case letters (Student-Newman-Keuls multiple comparison, $P<0.05$ ). aberrant growth. Some tubes were short, branching and with irregular tube wall growth (Fig. 3B). Other tubes became occluded by callose deposition at their tips soon after germinating (Fig. 3D).

Pollen germination in vitro was not affected by streptomycin added to the media at concentrations up to $400 \mathrm{mg} \cdot \mathrm{L}^{-1}$. Germination ranged from $53.7 \%$ to $60.3 \%$. Analysis of variance revealed no significant differences among treatments or between cultivars. Pollen tube growth in the streptomycin-
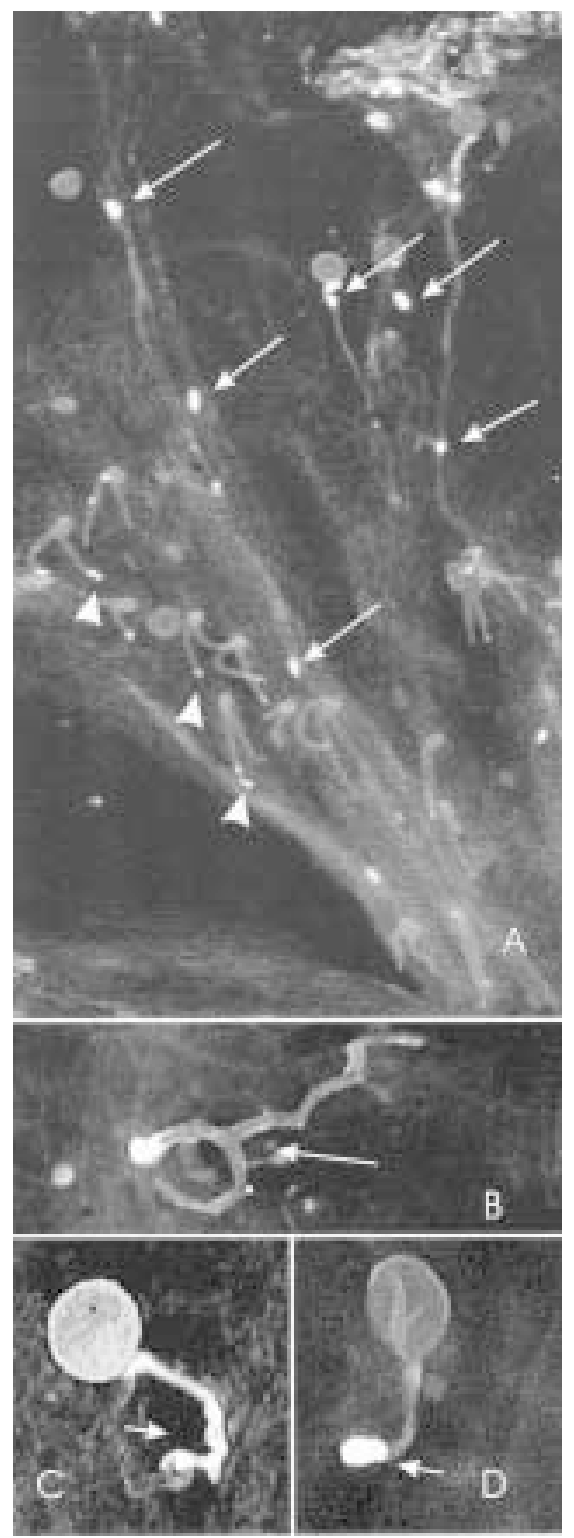

Fig. 3. Pollen tubes in stigma-style squashes. Squashes were stained with alkaline aniline blue, which demonstrates the presence of callose. (A) Untreated control. Pollen tubes are long, straight, unbranched and have regular callose plugs (arrows). Trichomes on the stylar surface also show callose at their tips (arrowheads). B, C, D. Pollen tubes from bloomperiod streptomycin treatment showing branching of tubes (B, arrow), irregular pollen tube wall formation (C, arrow), and pollen tubes whose growth has been stopped with tips that have become densely occluded by callose deposition soon after pollen germination ( $\mathbf{D}$, arrow). 
supplemented media showed no apparent retardation of growth nor was there any evidence of structural anomalies comparable to those seen in vivo.

\section{Discussion}

Walnut pistillate flowers have a two-lobed stigma, which is subtended by a short, sessile style. The large basal gynoecium includes an ovary with one locule that contains an orthotropous ovule. The ovule is enclosed by a single integument. Pollen tubes germinate on the stigma surface, grow through the stigma-style transmitting tract and enter the ovary. Pollen tube entry into the ovule may be porogamous (through the micropyle) or chalazogamous (through the base of the ovule at the chalaza) depending on the degree of integument development that had occurred when pollen tubes reach the ovule (Luza and Polito, 1991). Fertilization typically occurs within a week of pollination. Endosperm proliferation occurs rapidly, and the first division of the zygote occurs $\approx 3$ weeks later. Fruits grow rapidly during the weeks after bloom. Walnut is unusual in that fruit from unpollinated flowers or flowers with unfertilized ovules remain on the tree and appear to grow normally through 3-5 weeks after bloom (12-20 mm diameter), at which time they abscise (Pinney and Polito, 1983).

Our results indicate that bloom-period applications of streptomycin affect both pollen germination and pollen tube growth in vivo. Fewer germinated pollen grains are present at the stigmas of flowers sprayed with the antibiotic at prebloom and during the bloom period relative to unsprayed controls, and fewer pollen tubes grow to the ovary in these treatments (Fig. 2). For the bloomperiod treatment, only $15 \%$ of the flowers we examined had even one pollen tube that had grown through to the base of the style.

Abnormal ovule development is evident in bloom-period treated flowers collected prior to the onset of fruit abscission. Indications of ovule degeneration are apparent at the integuments and the nucellus. Endosperm is lacking and there is no proembryo present. These abnormalities are consistent with a failure of normal pollen tube growth to the ovule and a lack of fertilization.

Although pollen germination and pollen tube growth are strongly inhibited by streptomycin treatment in vivo, no effect is seen on streptomycin-supplemented media in vitro. As an aminoglycoside, streptomycin acts as an inhibitor of protein synthesis in prokaryotic-type ribosomes (Pestka, 1971), where it can cause misreading of mRNA during translation (Davies et al., 1964). Streptomycin can induce bleaching of eukaryotic plants (Bourque et al., 1976; Maliga et al., 1975; Mancinelli et al., 1975) and can inhibit the utrastructural development of plastids (Behn and Arnold, 1974). It is not unexpected to find that it does not affect pollen germination, where plastids do not play a role. It is possible that streptomycin acts to alter conditions at the stigma surface (as, for example, by affecting protein synthesis in plastids of stigmatic cells thereby disrupting stigma-cell events involving pollen-stigma interactions during pollen germination), rather than acting on pollen or pollen tubes directly.

Kimura et al. (1996) investigated the effect of streptomycin sprays on 'Muscat Bailey' grape (Vitis vinifera L. $\times$ V. labrusca L.). They found that it did not inhibit pollen germination or tube growth; however, it did lead to increased seedlessness.

A second possibility is that the streptomycin treatment affects pollen prior to its being shed from the catkins, rendering it incapable of normal germination and growth. We did not examine pollen germination and growth in vitro using pollen from the streptomycin treatments, so we cannot address this possibility directly. However, this does not seem to be a likely explanation for two reasons. First, walnut is anemophilous; thus, in an orchard situation vast amounts of airborne pollen are coming from other, nearby trees that did not receive the streptomycin treatment. This would provide an ample source of unaffected and presumably healthy pollen capable of germination, normal pollen tube growth, and fertilization. Furthermore, 'Vina' is a protandrous cultivar; as such, pollen and catkin development is advanced relative to pistillate flower development. If streptomycin does affect pollen maturation, then one should expect to see a greater effect from the prebloom treatment when pollen maturation was occurring in the subject trees.

Although the precise mechanism by which streptomycin affects pollen germination and tube growth is unclear, it is apparent that bloom-period streptomycin sprays lead to high levels of fruit drop by acting to inhibit pollen tube growth sufficiently to prevent fertilization of the ovule. Postbloom and prebloom spray treatments, which do not interfere with these processes or do so to a lesser extent, are potentially effective for control of $X$. campestris pv. juglandis.

\section{Literature Cited}

Behn W. and C.G. Arnold. 1974. The effect of streptomycin and neamine on the structure of chloroplast and mitochondria in Chlamydomonas reinhardi. Protoplasma 82:77-89.

Bourque, D.P., P.N. McMillan, W.J. Clingenpeel, and A.W. Naylor. 1976. Comparative effects of several inhibitors of chloroplast thylakoid membrane synthesis in greening jack bean. Bot. Gaz. 137:279-284.

Buchner, R.P., W.H. Olson, V.S. Polito, and K. Pinney. 2000. Effect of streptomycin walnut blight sprays on nut drop. HortScience 35:423. (Abstr.)

Davies, J., W. Gilbert, and L. Gorini. 1964. Streptomycin, suppression, and the code. Proc. Natl. Acad. Sci. 51:659-664

Kimura, P.H., G. Okamoto, and K. Hirano. 1996. Effects of gibberellic acid and streptomycin on pollen germination and ovule and seed development in Muscat Bailey A. Amer. J. Enol. Viticult. 47:152-156.

Luza, J.G. and V.S. Polito. 1987. Effect of desiccation and controlled rehydration on in vitro germination of walnut (Juglans spp.) pollen Plant, Cell Environ. 10:487-492.

Luza, J.G. and V.S. Polito. 1991. Porogamy and chalazogamy in walnut (Juglans regia L.). Bot. Gaz. 149:30-36.

Maliga, P., A.S. Brezonvits, and L. Marton. 1975. Non-Mendelian streptomycin-resistant tobacco mutant with altered chloroplasts and mitochondria. Nature 255:401-402.

Mancinelli, A.L., C.P.H. Yang, P. Lindquist, O.R. Anderson, and I. Rabino. 1975. Photocontrol of anthocyanin synthesis. III. The action of streptomycin on the synthesis of chlorophyll and anthocyanin. Plant Physiol. 55:251-257.

Pinney, K. and V.S. Polito. 1983. English walnut fruit growth and development. Sci. Hort. 21:1928.

Sokal, R.R. and Rohlf, F.J. 1995. Biometry. 3rd ed. W.H. Freeman and Co., N.Y.

Teviotdale, B.L. and M.N. Schroth. 1998. Bark, fruit, and foliage diseases, p. 242-246. In: D.E. Ramos (ed.). Walnut Production Manual. Publ. 3373. Div. Agr. Nat. Sci., Univ. of California, Oakland. 